\title{
Investigating Cortical Variability Using a Generic Gyral Model
}

\author{
Gabriele Lohmann ${ }^{1}$, D. Yves von Cramon ${ }^{1}$, and Alan C.F. Colchester ${ }^{2}$ \\ ${ }^{1}$ Max-Planck-Institute for Human Cognitive and Brain Sciences, Leipzig, Germany \\ ${ }^{2}$ University of Kent at Canterbury, UK
}

\begin{abstract}
In this paper, we present a systematic investigation of the variability of the human cortical folding using a generic gyral model (GGM). The GGM consists of a fixed number of vertices that can be registered non-linearly to an individual anatomy so that for each individual we have a clearly defined set of landmarks that is spread across the cortex. This allows us to obtain a regionalized estimation of intersubject variability. Since the GGM is stratified into different levels of depth, it also allows us to estimate variability as a function of depth. As another application of a polygonal line representation underlying the generic gyral model, we present a cortical parcellation scheme that can be used to regionalize cortical measurements.
\end{abstract}

\section{Introduction}

Human cortical folding shows a high degree of inter-individual variability which makes inter-subject comparisons very difficult and prone to error. In this paper, we systematically investigate inter-subject variability using a set of cortical landmarks. These landmarks are derived from a generic gyral model (GGM) that was previously introduced in [1]. The vertices of the GGM serve as landmarks that can be easily identified across a large population of subjects using non-linear registration techniques. Their variances and covariances can then be analyzed and used in a regionalized way.

The identification of useful landmarks is a key problem in inter-subject registration and thus in variability studies 2. We use the vertices of the generic gyral model because this model is very well justified as an average representation of cortical gyri across a large population of subjects.

As another extension of the work in [1] we present a novel cortical parcellation technique. While in our previous work, gyri were represented as polygonal lines, we now extend this concept so that entire gyral surfaces can be segmented and a semi-complete parcellation of the cortical surface results. This will be helpful for regionalizing cortical measurements such a thickness estimations.

\section{Parcellation of the Cortical Surface}

In 1], a polygonal line representation of cortical gyri was introduced. A polygonal line consists of a set of vertices in $3 \mathrm{D}$ space that are connected by edges. We 
envisage a thin 3D sheet residing at the centre of a gyrus that is obtained by a 3D topological thinning operation. A gyral line intersects this sheet at a userdefined level of depth (see figure 11). A set of such lines at increasing levels of depth provide a simplified representation of a gyral sheet.

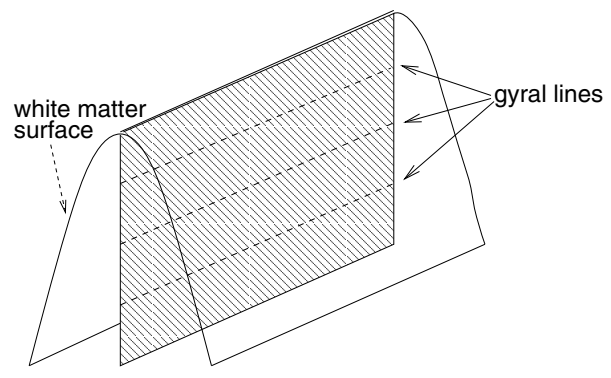

Fig. 1. A gyral line is a polygonal line the intersects the skeletonized gyrus (gyral sheet) at a given level of depth. In this paper, we investigate sets of gyral lines that intersect the gyral sheet at eight pre-defined depth levels.

A semi-automatic approach can be used to obtain neuroanatomical labels for some major gyri 1. Specifically, the following gyri can be identified in most individuals: inferior frontal, pre- and postcentral, perisylvian, temporal, and a parietal gyrus.

Here, this approach is extended so that entire gyri can be segmented and labelled and a parcellation of the cortical surface results. This parcellation does not cover the entire cortical surface as the gyral labelling is restricted to the major gyri. Figure 2 shows this method applied to one individual brain. As input into the algorithm, a skull-stripped T1-weighted image, its white matter segmentation and the corresponding labelled gyral line representation is required. For each voxel on the white matter surface, the nearest vertex of the gyral lines is sought. If this vertex has a neuroanatomical label then the white matter surface voxel inherits this label, otherwise it receives a null label. Once all white matter surface voxels are labelled in this manner, all grey matter voxels are labelled likewise: each grey matter voxel inherits the label of the nearest white matter voxel.

\section{Obtaining Cortical Landmarks}

In [1, a generic model of human cortical gyri was obtained using non-linear principal components analysis applied to 96 T1-weighted MRI data sets (see figure 4). The generic gyral model (GGM) consists of an averaged line representation for some major gyri at eight depth levels. Each depth level contains about 85 vertices so that the entire model has about 700 vertices. Here, we propose to use these vertices as landmarks for inter-subject registration. Since these landmarks can be identified in each individual via non-linear registration, they can 

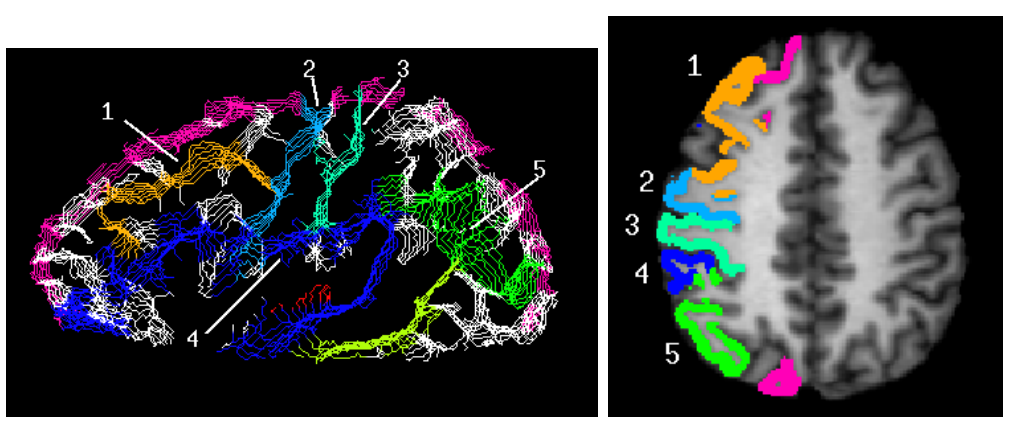

Fig. 2. Mapping gyral labels from the line-based representation to the cortical surface (1:middle frontal, 2: precentral, 3: postcentral, 4:perisylvian, 5: parietal)

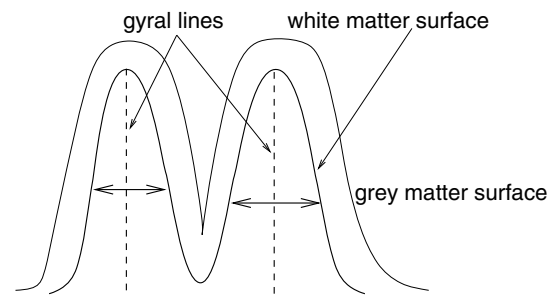

Fig. 3. Mapping of gyral labels from the line-based representation to the cortical surface: each point on the white matter surface inherits the label of the nearest gyral line, and each point within the grey matter inherits these labels

subsequently be used to describe and analyze inter-subject variability. In the following, we describe our non-linear inter-subject registration method.

As a prerequisite to any further processing, all MRI data sets are assumed to be registered to an $\mathrm{AC} / \mathrm{PC}$-based coordinate frame [3] using an affine linear transformation. The GGM can be then used to guide inter-subject registrations since it provides a set of cortical landmarks. In the following, we propose a two-stage approach: at the first stage, a cubic polynomial is applied to provide an initial rough match. The second stage employs thinplate splines for a more refined match.

Both stages require a point-to-point correspondence between the vertices of the GGM and the vertices of the gyral lines of an individual data set. However, the number of vertices differs greatly between these two types of data. The generic gyral model contains about 700 vertices across eight different levels of depth. Since the GGM is an averaged representation it is a lot smoother than the line representations of individual data. In addition, the GGM only contains neuroanatomically labelled major gyri, whereas line representations of individual data also contain numerous unlabelled smaller gyri. Therefore, the number of vertices of an individual data set is dramatically larger than that of the GGM.

To obtain point correspondences, we have tested several methods including the assignment method by Rangarajan et al. 4] which worked quite well. However, 


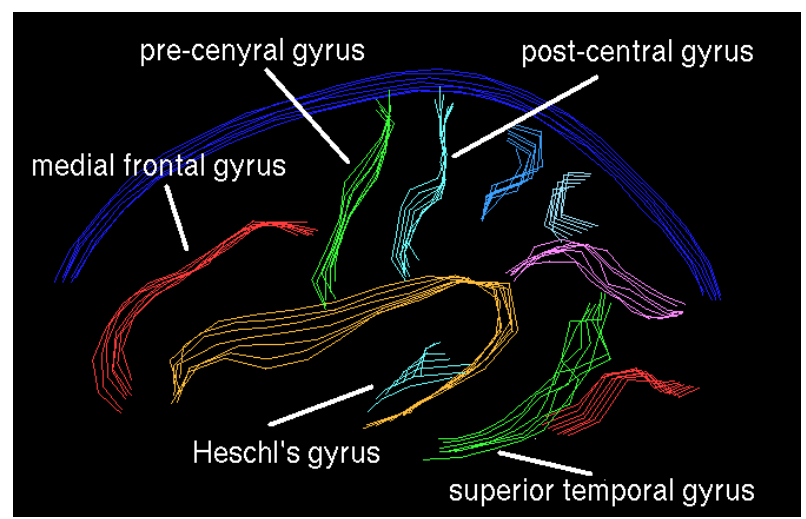

Fig. 4. The generic gyral model (see [1])

we found that a simple nearest neighbour assignment produced similar results where for each point $p_{i}, i=1, \ldots, n$ of the GGM, the gyral line point $q_{j}$ with minimal Euclidean distance and the same neuroanatomical label is selected. For an initial rough match, a cubic polynomial of the following form is used:

$$
\begin{aligned}
f(x, y, z)= & a_{1} x+a_{2} y+a_{3} z+a_{4} x y+a_{5} x z+a_{6} y z+a_{7} x^{2}+a_{8} y^{2}+a_{9} z^{2} \\
& +a_{10} x y z+a_{11} x^{2} y+a_{12} x^{2} z+a_{13} y^{2} x+a_{14} y^{2} z+a_{15} z^{2} x+a_{16} z^{2} y \\
& +a_{17} x^{3}+a_{18} y^{3}+a_{19} z^{3} .
\end{aligned}
$$

The deformation parameters $a_{1}, \ldots, a_{19}$ are estimated such that the term

$$
\sum_{i, j}\left(q_{j}-f\left(a_{1}, \ldots, a_{19} ; p_{i}\right)\right)^{2}
$$

is minimized. In our experiments, we have used Powell's method [5] for non-linear optimization. This initial registration is then refined using a thinplate spline approach 67]. As for the cubic polynomial, the point-to-point correspondence is obtained by selecting for each point $p_{i}, i=1, \ldots, n$ of the GGM a gyral line point $q_{j}$ with minimal Euclidean distance and the same neuroanatomical label. Since the cubic polynomial was applied beforehand, the Euclidean distances are now much smaller than before. Note that the cubic polynomial is governed by global parameters that minimize the distances between the two data sets globally, whereas the thinplate splines produce a local match that moves each landmark point directly onto its nearest corresponding point.

\section{Regionalized Estimation of Cortical Variability}

As described above, the GGM (and hence the vertices) can be registered nonlinearly so that for each individual we have a fixed number of landmarks at defined locations spread across the cortex at various levels of depth. This allows us to obtain a regionalized estimation of inter-subject variability. Since the GGM 
is stratified into different levels of depth, it also allows us to estimate variability as a function of depth.

For each of the 96 data sets, we extracted a 3D gyral line representation and performed a non-linear registration that mapped the vertices of the GGM onto each individual data set using the method described in the previous section. This yielded a deformation map that contained a displacement vector for each GGM-vertex in each individual. For each individual, we obtained a map that recorded the magnitude of the displacement (see figure 5).

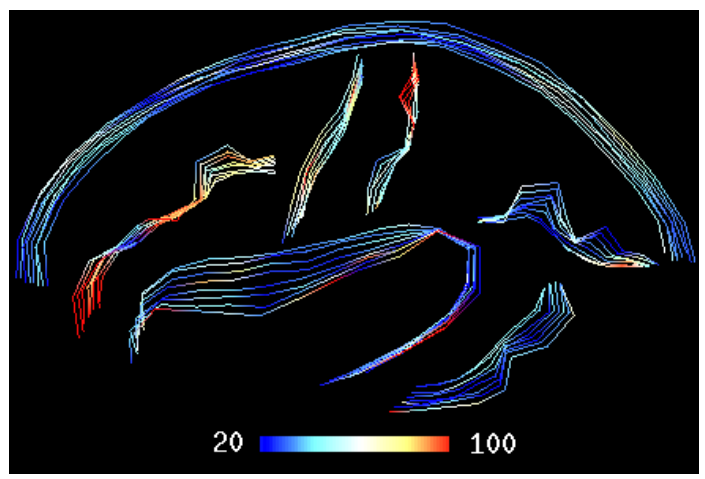

Fig. 5. A displacement map of one individual data set. The displacement is corrected for the bias introduced by the $\mathrm{AC} / \mathrm{PC}$-alignment by dividing the displacement magnitude by the distance from $\mathrm{AC} / \mathrm{PC}$ (see text). Therefore, the color-coded values correspond to corrected displacement magnitudes.

Note however that such a measure of displacement is biased by the initial $\mathrm{AC} / \mathrm{PC}$ alignment (see figure 6). Landmarks that are closer to $\mathrm{AC} / \mathrm{PC}$ will tend to be less variable than those that are further away. To correct for this problem, we divided the displacement magnitude of each vertex by its distance from $\mathrm{AC} / \mathrm{PC}$.

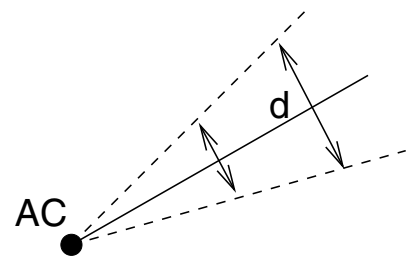

Fig. 6. Measures of variability must be corrected for the bias introduced by the AC/PC alignment

The displacements record the deviation from the generic gyral model. Since the model represents a population average, large displacements over many subjects indicate a high degree of variability. Therefore, the individual displacement 
maps were averaged across the 96 data sets to produce a map that represents the variability across the population (see figure 7). Note that the middle frontal gyrus shows a higher degree of variability than the parietal gyrus. Also note that the dorsal tip of the pre- and postcentral gyri is more variable than their ventral parts.
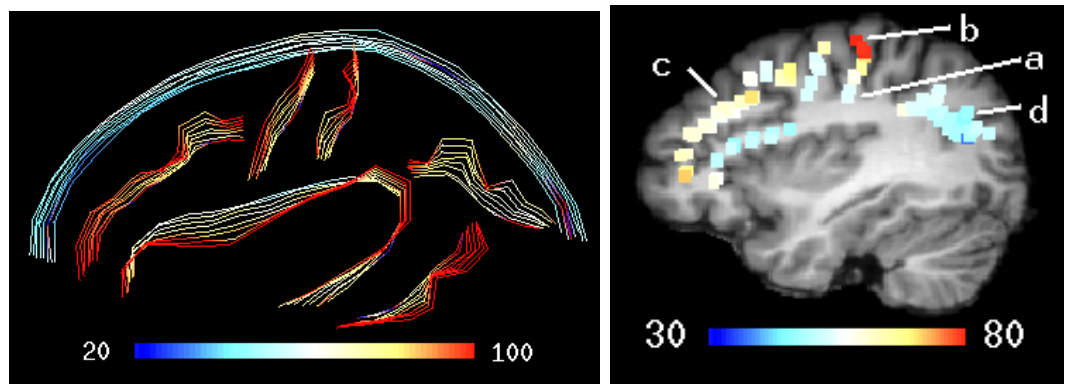

Fig. 7. Variability map computed as an average of displacement maps of 96 subjects. The right-hand image shows the vertices of the GGM superimposed onto a T1-weighted structural image. The ventral region of the post-central gyrus (a) is less variable than the dorsal region (b). The parietal gyrus (d) is less variable than the middle frontal gyrus (c). The color coding is based on the AC/PC-corrected variability measure described in the text.

Since the GGM contains eight different strata of depth, we can investigate the inter-subject variability as a function of depth. For this purpose, we extracted a deep and a shallow level of depth and performed non-linear registrations separately for both levels. We then extracted the main gyri contained in the GGM for both depth levels across all 96 subjects.

We estimated the standard deviation in $x, y, z$ for each landmark and divided this estimate by the distance from the landmark to the anterior commissure (AC). This was done to counterbalance the bias in variance estimation introduced by the $\mathrm{AC} / \mathrm{PC}$-alignment. We found that in 5 out of 6 main gyri, the variance decreased with depth when comparing the deepest level against the most shallow level. Table 1 shows the results. Note that comparisons between gyri are not possible with this approach as the gyri are represented by a different number of vertices.

Table 1. The decrease in variance with depth. The estimates correspond to standard deviations averaged across the landmarks within a gyrus and summed over $x, y, z$. To account for the bias in estimating variances, the standard deviations were divided by the distance from anterior commissure (AC) for each landmark separately.

\begin{tabular}{|l|c|c|c|c|c|c|}
\hline & perisylvian & precentral & postcentral & parietal & temporal & middle frontal \\
\hline deep & 6.890 & 2.689 & 1.811 & 2.435 & 6.054 & 5.399 \\
shallow & 8.892 & 3.837 & 1.995 & 2.128 & 6.085 & 5.578 \\
\hline
\end{tabular}




\section{Discussion}

We have presented a new method for analyzing cortical variability and for obtaining cortical parcellation. Some previous methods for analyzing the variability were also based on extracting labelled representations of sulci or gyri (e.g. 869]). The novelty of our approach lies the usage of the generic gyral model which provides a set of well-defined landmarks that can be identified across may subjects using non-linear registration. These landmarks are particularly useful because they are located on main gyri that can be easily detected in most healthy subject. Also, these landmarks are separated into different strata of depth so that variability can be investigated as a function of depth. We have found that variability decreases with depth which agrees well with earlier findings 1011.

While our approach is essentially line-based (or vertex-based), several other authors have used surface-based approaches for analyzing cortical variability (e.g. 122]). Van Essen [2] however noted the importance of identifying useful landmarks. Here, we have proposed to use landmarks that are derived from the generic gyral model. These landmarks are very well justified because the generic gyral model resulted from averages across a large population of individual data sets. Therefore, we can expect these landmarks to be quite representative of major cortical features.

One aspect of our future work will be directed towards exploiting the parcellation of the cortical surface so that morphometric studies can be regionalized.

\section{References}

1. G. Lohmann,D.Y. von Cramon, A.C.F. Colchester: A construction of an averaged representation of human cortical gyri using non-linear principal component analysis. In: 8th Int. Conf. on Medical Image Computing and Computer Assisted Intervention (MICCAI 2005), Palm Springs, CA, USA (2005)

2. Essen, D.V.: A population-average, landmark- and surface-based (pals) atlas of the human cerebral cortex. Neuroimage 28 (2005) 635-662

3. Talairach, P. Tournoux, J.: Co-planar Stereotaxic Atlas of the Human Brain: 3Dimensional Proportional System - an Approach to Cerebral Imaging. Thieme Medical Publishers, New York (1988)

4. Gold, A. Rangarajan, S.: A graduated assignment algorithm for graph matching. IEEE Transactions on Pattern Analysis and Machine Intelligence 18 (1996)

5. Press, W.H., Teukolsky, S.A., Vetterling, W.T., Flannery, B.P.: Numerical Recipes in C. 2nd edn. Cambridge University Press (1992)

6. Bookstein, F.: Principal warps: thin-plate splines and the decomposition of deformations. IEEE Transactions on Pattern Analysis and Machine Intelligence 11 (1989) 567-585

7. Bookstein, F.: Morphometric tools for landmark data. Cambridge University Press (1991)

8. Goualher, E. Procyk, D.L. Collins, R. Venugopal, C.Barillot, A.C. Evans, G.L.: Automated extraction and variability analysis of sulcal neuroanatomy. IEEE Transactions on Medical Imaging 18 (1999) 206-217 
9. Cachia, J.F. Mangin, D. Riviere, F. Kherif, N. Boddaert, A.Andrade, D. Papadopoulos-Orfanos, J.B. Poline, I. Bloch, M. Zilbovicius, P. Sonigo, F. Brunelle, J. Regis, A.: A primal sketch of the cortex mean curvature: A morphogenesis based approach to study the variability of the folding patterns. IEEE Transactions on Medical Imaging 22 (2003) 754-765

10. G.Lohmann, D.Y.v.Cramon, H. Steinmetz: Sulcal variability of twins. Cerebral Cortex 9 (1999) 754-763

11. Goualher, A.M. Argenti, M. Duyme, W.F.C. Baar, H.E. Hulshoff Pol, D.I. Boomsma, A. Zouaoui, C. Barillot, A.C. Evans, G.L.: Statistical sulcal shape comparisons: application to the detection of the genetic encoding of the central sulcus shape. Neuroimage 11 (2000) 564-574

12. Thompson, T.D. Cannon, A.W. Toga, P.: Mapping genetic influences on human brain structure. Annals of Medicine 34 (2002) 523-536 